\title{
Producción y composición de la hojarasca en un renoval pre andino de Nothofagus glauca de la región del Maule
}

\author{
Litter production and composition in a young pre-Andean \\ Nothofagus glauca forest of Maule Region
}

\author{
Ursula Doll $^{\text {a*}}$, Pablo Araya ${ }^{\text {b, Luis Soto-Cerda a , Darío Aedo a , Gabriela Vizcarra }}{ }^{\text {c }}$ \\ *Autor de correspondencia: ${ }^{a}$ Universidad de Talca, Facultad de Ciencias Forestales, Talca, Chile, \\ Casilla 721-747, udoll@utalca.cl \\ ${ }^{\mathrm{b}}$ Universität Freiburg, Fakultät für Umwelt und Natürlichen Ressourcen, Deutschland. \\ c Universidad Católica del Maule, Talca, Chile.
}

\begin{abstract}
SUMMARY
With the aim of advancing in the comprehension of Maulino forest functioning, the annual litter production and its specific composition in a young pre-Andean Maulino forest dominated by hualo (Nothofagus glauca) were estimated. For this, 30 litter traps of $0.25 \mathrm{~m}^{2}$ were placed in three lines perpendicular to the slope on the floor of the forest. The litter was collected at variable intervals and separated into different components. During the three evaluated seasons, the annual mean production was $7.13 \mathrm{Mg}^{-1}$, of which $46 \%$ was leaves and $21 \%$ woody material. In two seasons, the other $33 \%$ was separated into leaves of different species. Thirty-one species were found, 15 of them endemic and two introduced. Leaves abscission of hualo principally concentrated during the winter season, whereas leaf fall of companion perennial species occurred during the summer months.
\end{abstract}

Key words: Nothofagus glauca, young pre-Andean forest, litter production, companion species.

\section{RESUMEN}

Con el fin de avanzar en la comprensión del funcionamiento del bosque maulino, se estimó la producción anual de hojarasca y su composición específica en un renoval pre andino dominado por hualo (Nothofagus glauca). A tal fin se instalaron 30 trampas de $0,25 \mathrm{~m}^{2}$ en tres líneas perpendiculares a la pendiente sobre el piso del renoval. En intervalos variables se cosechó el material producido, separándolo en sus distintos componentes. Durante tres temporadas, se cuantificó una producción promedio anual de $7,13 \mathrm{Mg}^{-1}$ (46\% de hojas de hualo y $21 \%$ de material leñoso). En dos temporadas, el $33 \%$ restante fue separado en hojas según especies, diferenciándose 31 especies ( 15 endémicas y dos introducidas). La abscisión de hojas de hualo se concentró principalmente en la época invernal, la caída de hojas de las especies acompañantes de carácter perennifolio ocurrió durante los meses estivales.

Palabras clave: Nothofagus glauca, renoval pre andino, producción de hojarasca, especies acompañantes.

\section{INTRODUCCIÓN}

Para predecir la respuesta del bosque frente a cambios climáticos globales es necesario conocer su funcionamiento (Ngao et al. 2005), siendo la productividad primaria un proceso fundamental del mismo. Tradicionalmente se estimó la producción de hojarasca con el fin de simular la productividad primaria neta en ecosistemas forestales $(\mathrm{Ca}-$ ritat et al. 2006) y para predecir la disponibilidad de carbono orgánico en el suelo (Wutzler y Mund 2007). La producción de hojarasca y su descomposición son esenciales para la transferencia de energía y nutrientes hacia el suelo (Diaz-Maroto et al. 2006), constituyéndose en un flujo que suple una importante fracción de nutrientes rápidamente mineralizables en bosques deciduos (Baum et al. 2009).

Mientras que la variabilidad interanual en la producción de hojarasca en bosques dominados por especies deciduas es directamente influenciada por las condiciones climáticas reinantes, en bosques de especies perennifolias la caída de hojarasca es menor y no refleja en forma precisa fluctuaciones inter anuales de la producción primaria, pero probablemente sea un buen predictor de su producción primaria promedio (Bellot et al. 1992, Lebret et al. 2001).

El hualo (Nothofagus glauca (Phil.) Krasser) es un árbol endémico y representativo de la región mediterránea de 
Chile, distribuido en ambas cordilleras debajo de $1.200 \mathrm{~m}$ s.n.m., entre $34^{\circ} 01^{\prime}$ S y $37^{\circ} 27^{\prime}$ S (Le-Quesne y Sandoval 2001). Debido a una fuerte extracción y sustitución por cultivos anuales y plantaciones exóticas, actualmente la especie está restringida a poblaciones sujetas a un intenso proceso de fragmentación, lo que gatilló su clasificación en la categoría de casi amenazada (MMA 2011).

El avance de las condiciones de aridez en el centro y sur de Chile (Stolpe y Undurraga 2016) impone una nueva restricción a la supervivencia de la especie. Altmann (2013) evaluó la relación entre el daño por insectos y el estado hídrico de hualo en distintas situaciones ambientales, concluyendo que el aumento de condiciones de aridez impactaría negativamente la especie, siendo mayor el riesgo para sus poblaciones más septentrionales $\left(34^{\circ} \mathrm{S} 71^{\circ} \mathrm{O}\right)$. Nothofagus macrocarpa (DC.) Vazq. et Rodr., especie afín de la región mediterránea, mostró un fuerte decaimiento del crecimiento radial entre 1980 y 2014, constatando gran sensibilidad a la variabilidad climática regional y global (Venegas-Gonzáleza et al. 2018). Fitzroya cupressoides (Mol.) Johnst., especie longeva del los bosques templado lluviosos del sur de Chile, evidenció una baja aclimatación a condiciones de aridez comparada con especies de Nothofagus de hoja perenne que, modificando la conductancia estomática, mejoraron la eficiencia en el uso del agua (Camarero y Fajardo 2017).

De $N$. glauca, cuya posición fitosociológica, distribución y asociación con comunidades vegetales fue detalladamente documentada (Amigo et al. 2000, Amigo y Rodríguez-Guitián 2011), existe escasa información sobre aspectos funcionales de sus comunidades. En este contexto, el objetivo del presente trabajo es determinar la producción de hojarasca y su composición temporal en un renoval de hualo de la precordillera andina.

\section{MÉTODOS}

Área de estudio. El muestreo de hojarasca se realizó en un renoval de hualo en el Centro Experimental "El Picazo", ubicado en la precordillera andina de la Comuna de San Clemente, región del Maule ( $35^{\circ} 31^{\prime} 19^{\prime \prime}$ y y $71^{\circ} 08^{\prime} 45^{\prime \prime O}$ ). Para la caracterización climática se utilizaron los registros

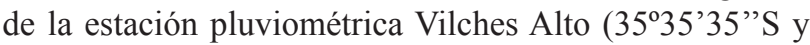
$71^{\circ} 05^{\prime} 13^{\prime \prime}$ ) $)$ y de la estación Meteorológica Colorado

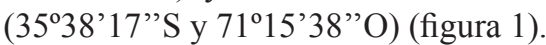

El clima templado cálido de tipo mediterráneo, se caracteriza por temperaturas mínimas entre 2 y $3{ }^{\circ} \mathrm{C}$ en la
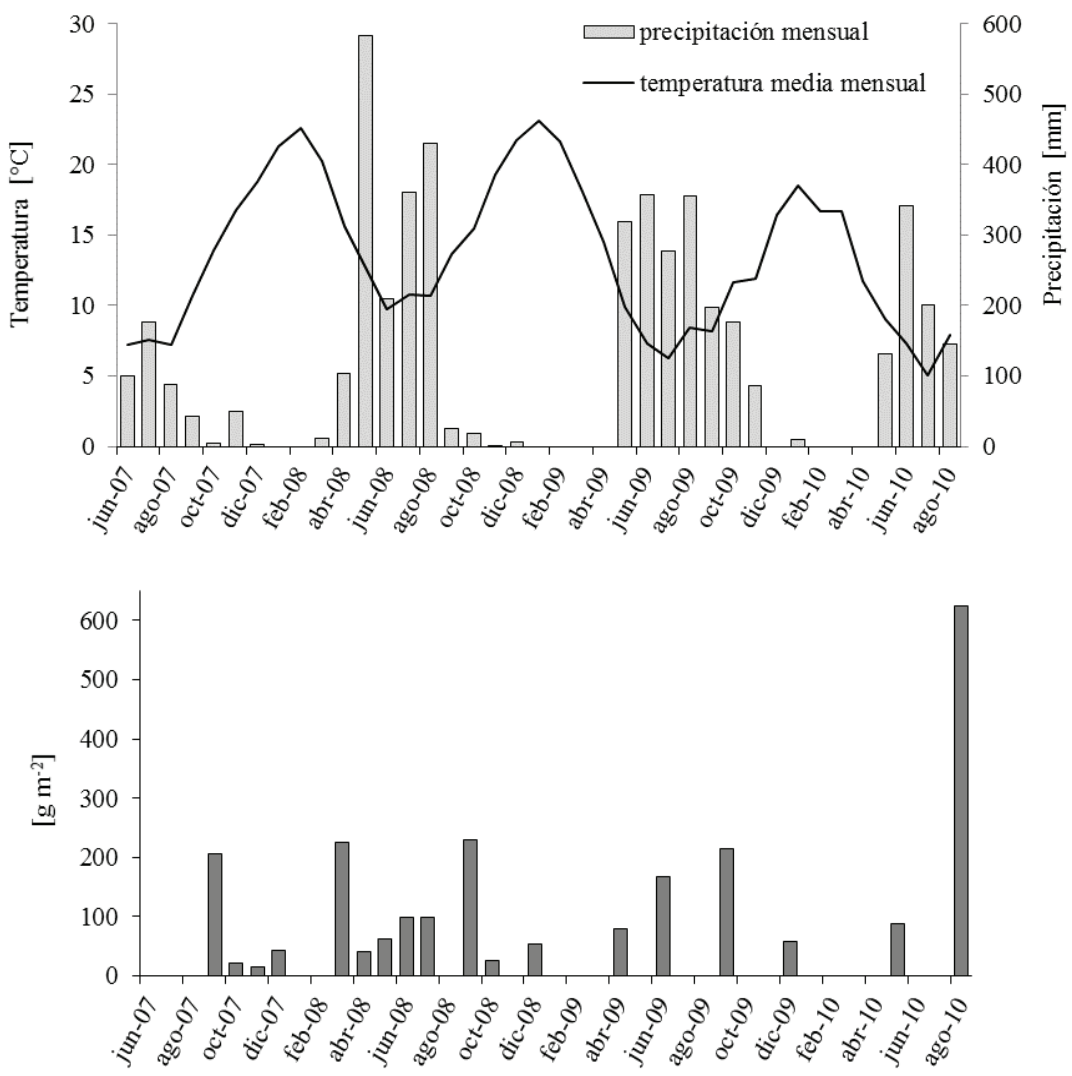

Figura 1. Precipitación mensual $[\mathrm{mm}]$, temperatura media mensual $\left[{ }^{\circ} \mathrm{C}\right]$ y producción de hojarasca total $\left[\mathrm{g} \mathrm{m}^{-2}\right]$ en un renoval pre andino de Nothofagus glauca de la región del Maule.

Monthly precipitation $[\mathrm{mm}]$, mean monthly temperature $\left[{ }^{\circ} \mathrm{C}\right]$ and total litter production $\left[\mathrm{g} \mathrm{m}^{-2}\right]$ in a young pre-Andean Nothofagus glauca forest of Maule Region. 
época invernal y máximas entre 23 y $27^{\circ} \mathrm{C}$ en la época estival. Las precipitaciones que se concentran entre los meses de mayo y septiembre promedian los $1.700 \mathrm{~mm}$ anuales. La topografía es montañosa, con pendientes moderadas a pronunciadas ( 0 a $30 \%$ ) y altitudes que fluctúan entre 400 y $900 \mathrm{~m}$. Predominan los suelos formados sobre cenizas volcánicas modernas, con textura franca a franco arcillosa en superficie, mediana pedregosidad y erosión moderada.

El renoval muestreado se ubicó a 700 m s.n.m., en una ladera de pendiente pronunciada ( $48 \%$ ) y exposición Sur. El estrato arbóreo estaba compuesto principalmente por individuos de hualo, de 14 a $17 \mathrm{~m}$ de altura, con densidad de 750 árboles ha ${ }^{-1} \mathrm{y}$ área basal de $21,10 \mathrm{~m}^{2} \mathrm{ha}^{-1}$, junto a 200 individuos ha-1 de otras especies con 3,67 $\mathrm{m}^{2} \mathrm{ha}^{-1} \mathrm{de}$ área basal. El suelo que sustenta el rodal es poco profundo, encontrándose el estrato rocoso a partir de $10520 \mathrm{~cm}$ de profundidad. La textura es franco arcillo arenosa, el contenido de fósforo Olsen $5 \mathrm{mg} \mathrm{kg}^{-1}$, posee cerca de un $10 \%$ de materia orgánica y una capacidad de intercambio catiónico de $37 \mathrm{cmol}+\mathrm{kg}^{-1}$ con $25 \%$ de saturación de bases.

Muestreo y procesamiento de hojarasca. Para la recolección de hojarasca se construyeron trampas de madera de $50 \mathrm{x}$ $50 \mathrm{~cm}$ y $10 \mathrm{~cm}$ de alto, cerradas en su base por una malla plástica (Raschel $65 \%$ sombra). En junio de 2007 se instalaron 30 trampas sobre el piso del renoval, en tres líneas perpendiculares a la pendiente. La distancia entre líneas fue $30 \mathrm{~m}$, ubicándose la primera a $30 \mathrm{~m}$ del borde superior del rodal. La posición inicial de las trampas en cada línea fue fijada al azar, disponiéndose 10 trampas equidistantes a $5 \mathrm{~m}$ sobre cada línea. Las trampas fueron colocadas en posición horizontal, ayudándose de troncos y ramas presentes en el piso del bosque. En intervalos variables, durante tres temporadas, se recolectó todo el material contenido en cada una de las trampas. En la última cosecha se descartaron siete trampas de las 30, por encontrarse sepultadas bajo troncos y ramas caídas a consecuencia de un temporal de nieve.

El material cosechado en cada trampa fue separado en hojas de hualo, material leñoso (ramas y corteza) y resto (hojas de otras especies, piezas florales, frutos, semillas y material no identificado). A partir de septiembre de 2008, el compartimento resto se separó a su vez en hojas de distintas especies y material no identificado. El material clasificado para cada trampa fue secado en estufa a $70{ }^{\circ} \mathrm{C}$ y pesado posteriormente con balanza de precisión.

\section{RESULTADOS}

Durante las tres temporadas evaluadas, se cuantificó una producción promedio anual de hojarasca de 713,4 \pm $152,4 \mathrm{~g} \mathrm{~m}^{-2}$, de la cual el $46 \%$ correspondió a hojas de hualo, $21 \%$ a material leñoso y $33 \%$ al resto. La composición de la hojarasca en distintos compartimentos mostró una marcada estacionalidad, que se repitió durante las tres temporadas consideradas. Entre los meses de octubre y diciembre de cada temporada se registró la menor producción de hojarasca, mientras que entre junio y septiembre se alcanzó la mayor producción (cuadro 1). En la colecta de

Cuadro 1. Producción de hojarasca $\left[\mathrm{g} \mathrm{m}^{-2}\right]$ separada en hojas de hualo, material leñoso y resto de la hojarasca, durante tres temporadas en un renoval pre andino de Nothofagus glauca de la región del Maule.

Litter $\left[\mathrm{g} \mathrm{m}^{-2}\right]$ separated into hualo leaves, branches + bark and rest of litter produced during three seasons in a young pre-Andean Nothofagus glauca forest of Maule Region.

\begin{tabular}{lccc}
\hline \multicolumn{1}{c}{ Fecha } & Hojas de hualo $\left[\mathrm{g} \mathrm{m}^{-2}\right]$ & Material leñoso $\left[\mathrm{g} \mathrm{m}^{-2}\right]$ & Resto $\left[\mathrm{g} \mathrm{m}^{-2}\right]$ \\
\hline Septiembre 2007 & $167,54 \pm 84,85$ & $19,55 \pm 14,02$ & $19,38 \pm 17,70$ \\
Octubre 2007 & $2,39 \pm 2,35$ & $5,61 \pm 9,52$ & $12,34 \pm 21,80$ \\
Noviembre 2007 & $2,43 \pm 1,45$ & $4,78 \pm 2,96$ & $6,99 \pm 3,40$ \\
Diciembre 2007 & $1,95 \pm 1,77$ & $2,29 \pm 2,76$ & $39,20 \pm 33,20$ \\
Marzo 2008 & $32,07 \pm 24,12$ & $32,48 \pm 24,98$ & $160,04 \pm 109,39$ \\
Abril 2008 & $10,86 \pm 12,90$ & $6,59 \pm 9,51$ & $22,45 \pm 14,56$ \\
Mayo 2008 & $22,07 \pm 14,80$ & $11,81 \pm 6,71$ & $28,25 \pm 18,50$ \\
Junio 2008 & $44,85 \pm 23,74$ & $22,42 \pm 9,31$ & $30,75 \pm 15,15$ \\
Julio 2008 & $50,81 \pm 29,91$ & $21,89 \pm 14,28$ & $25,19 \pm 15,77$ \\
Septiembre 2008 & $185,64 \pm 82,23$ & $23,95 \pm 32,07$ & $19,56 \pm 26,27$ \\
Octubre 2008 & $3,59 \pm 3,12$ & $6,03 \pm 10,09$ & $16,03 \pm 18,45$ \\
Diciembre 2008 & $2,50 \pm 2,86$ & $6,08 \pm 11,36$ & $44,01 \pm 35,98$ \\
Abril 2009 & $13,05 \pm 11,54$ & $8,40 \pm 12,17$ & $58,73 \pm 44,47$ \\
Junio 2009 & $111,19 \pm 49,08$ & $19,41 \pm 25,47$ & $36,65 \pm 23,52$ \\
Septiembre 2009 & $165,49 \pm 78,31$ & $35,12 \pm 34,79$ & $14,59 \pm 7,82$ \\
Diciembre 2009 & $8,90 \pm 4,26$ & $18,22 \pm 19,06$ & $30,15 \pm 20,39$ \\
Mayo 2010 & $7,04 \pm 4,16$ & $7,44 \pm 7,46$ & $73,77 \pm 68,01$ \\
Agosto 2010 & $314,33 \pm 122,78$ & $214,74 \pm 112,56$ & $93,91 \pm 46,95$ \\
\hline
\end{tabular}


marzo 2008 también se obtuvo un máximo de producción de hojarasca que consistió principalmente en material procedente de especies perennifolias, mientras que los picos de producción de agosto-septiembre correspondían en su mayoría a hojas de hualo. La hojarasca producida durante el verano de las dos últimas temporadas registradas también estuvo compuesta principalmente por material procedente de especies de hoja perenne.

En la línea de trampas más cercanas al borde del rodal se acumuló menos material del compartimento resto. Durante el invierno 2010 la acumulación de nieve provocó la caída de muchos árboles después de una fuerte nevada, que se tradujo en un aumento del material leñoso acumulado en las trampas.

El compartimento resto lo integraron 31 especies, de las cuales 15 son endémicas y dos introducidas (anexo 1). Entre las especies acompañantes del renoval, se destacaron las arbóreas siempreverdes peumo (Cryptocarya alba (Molina) Looser), litre (Lithraea caustica (Molina) Hook. et Arn.), arrayán (Luma apiculata (DC.) Burret), boldo (Peumus boldus Molina), la especie invierno verde maqui (Aristotelia chilensis (Molina) Stuntz) y los arbustos siempreverdes Azara integrifolia Ruiz et Pav., Escallonia pulverulenta (Ruiz et Pav.) Pers. y Myrcengenia obtusa (DC.) O. Berg. Estas especies sumaron en conjunto el $59 \%$ del compartimento resto, concentrando su caída de follaje en la época estival. Las otras especies, que contribuyeron con un $14 \%$ al resto, presentaron el período de abscisión más tardío. En este último grupo destacaron Boquila trifoliolata (DC.) Decne. y Lardizabala biternata Ruiz et Pav., dos especies trepadoras, y Pinus radiata D. Don (pino), aportado por el viento de una plantación contigua al bosque nativo. Las acículas de pino se encontraron concentradas principalmente en la línea más cercana al límite del renoval colindante con la plantación.

\section{DISCUSIÓN}

La producción de hojarasca anual promedio estimada para el renoval pre andino de hualo $\left(7,13 \mathrm{Mg} \mathrm{ha}^{-1}\right)$, es inferior a la obtenida en un año por Palacios-Bianchi (2002), para un rodal costero de la región dominado por hualo $\left(8,36 \mathrm{Mg} \mathrm{ha}^{-1}\right)$. Sin embargo, la producción de la primera temporada, con $8,30 \mathrm{Mg} \mathrm{ha}^{-1}$, se acerca a este valor, a diferencia de la producción promedio de $6,55 \mathrm{Mg} \mathrm{ha}^{-1}$ alcanzada en las dos siguientes temporadas.

Es probable que parte del material acumulado en las trampas durante la segunda y tercera temporada se perdiera por descomposición, debido a la menor periodicidad con que se recolectó la hojarasca. En el bosque costero se perdió más del $10 \%$ del peso inicial de hojas de hualo vía descomposición durante los primeros 83 días, disminuyendo posteriormente su tasa de descomposición (Palacios-Bianchi 2002). También es de esperar una menor producción de hojarasca en el caso del renoval pre andino más joven en comparación con el bosque maduro costero. En rodales de
Fagus sylvatica L. se observó un aumento de la producción de hojarasca con la edad del bosque (Lebret et al. 2001).

La estacionalidad en la composición de la hojarasca, en distintos compartimentos y en cada temporada, es una característica registrada en la mayoría de los bosques. Mientras que la abscisión de hojas de hualo se concentró en la época invernal, al igual que en otras especies caducifolias de clima mediterráneo (Gallardo et al. 1998), las especies acompañantes perennifolias perdieron sus hojas durante el período estival (Bellot et al. 1992, Caritat et al. 2006, Martínez-Alonso et al. 2007). A diferencia del bosque costero, donde se concentró la abscisión de hojas de hualo a comienzos del invierno (junio), en el renoval pre andino ocurrió al final del invierno (agosto), discrepancia debida posiblemente al clima predominante en cada sitio.

Las condiciones de extrema aridez del verano 20072008, probablemente favorecieron la mayor abscisión de hojas de las especies perennifolias, cuya estrategia permite disminuir la tasa transpiratoria. Durante las dos temporadas anteriores (2005-2006), las precipitaciones superaron la media anual con 2.371 y $2.180 \mathrm{~mm}$, respectivamente, lo que posiblemente indujo una mayor acumulación de biomasa en las especies de hoja perenne o una mayor retención de hojas antiguas. Caritat et al. (2006) comprobaron que los años con mayor producción corresponden a los más calurosos. La caída de material leñoso se comportó de forma más errática que los otros componentes, asociada a las condiciones de ventosidad y al temporal de nieve (Caritat el al. 2006).

La mayor acumulación del compartimento resto en las trampas más alejadas del borde del bosque se relaciona a un arrastre de este material pendiente abajo y a una mayor presencia de especies distintas de hualo al interior del bosque. Vilà et al. (2004) encontraron que existe una mayor acumulación de mantillo en bosques integrados por varias especies que en bosques mono específicos.

La mitad de las especies que aportaron a la producción de hojarasca son endémicas (anexo 1). Esta situación evidencia la riqueza del bosque maulino, que es hábitat y refugio para diversos taxa, muchos de los cuales se encuentran en distintos grados de amenaza. Debido a los procesos de fragmentación que actualmente ocurren en estos bosques y las proyecciones relacionadas al cambio climático, la generación de conocimiento acerca de la dinámica de estos bosques es relevante para describir su comportamiento como sistema y para comprender aspectos relativos a su capacidad de respuesta ante cambios en las condiciones ambientales. Frente al aumento de las condiciones de aridez, las diferentes especies manifestarán capacidades de ajuste específicas, que se traducirán en un desplazamiento de las especies menos tolerantes.

\section{REFERENCIAS}

Altmann S. 2013. Crown condition, water availability, insect damage and landscape features: are they important to the Chilean tree Nothofagus glauca (Nothfagaceae) in the con- 
text of climate change? Australian Journal of Botany 61: 394-403.

Amigo J, A Rodríguez-Guitián. 2011. Bioclimatic and phytosociological diagnosis of the species of the Nothofagus genus (Nothofagaceae) in South America. Internatioanl Journal of Geobotanical Research 1: 1-20.

Amigo J, J San Martín, L García. 2000. Estudio fitosociológico de los bosques de Nothofagus glauca (Phil.) Krasse del Centro-Sur de Chile. Phytocoenologia 30(2): 193-221.

Baum C, M Fienemann, S Glatzel, G Gleixner. 2009. Overstoryspecific effects of litter fall on the microbial carbon turnover in a mature deciduous forest. Forest Ecology and Management 258: 109-114.

Bellot J, J Sánchez, M. Lledó, P Martínez, A Escarré. 1992. Litterfall as a measure of primary production in Mediterranean holm-oak forest. Vegetatio 99-100: 69-76.

Camarero J, A Fajardo. 2017. Poor acclimation to current drier climate of the long-lived tree species Fitzroya cupressoides in the temperate rainforest of southern Chile. Agricultural and Forest Meteorology 239: 141-150.

Caritat A, E García-Berthou, R Lapeña, L Vilar. 2006. Litter production in a Quercus suber forest of Montseny (NE Spain) and its relationship to meteorological conditions. Annals of. Forest Science 63:791-800.

Diaz-Maroto I, P Vila-Lameiro. 2006. Litter production and composition in natural stands of Quercus robur L. (Galicia, Spain). Polish journal of Ecology 54(3): 429-439.

Gallardo J, A Martin, I Santa Regina. 1998. Nutrient cycling in deciduous forest ecosystems of the Sierra de Gata mountains: aboveground litter production and potential nutrient return. Annals of Forest Science 55: 749-769.

Lebret M, C Nys, F Forgeard. 2001. Litter production in an Atlantic beech (Fagus sylvatica L.) time sequence. Annals of Forest Science 58: 755-768.

Le-Quesne C, L Sandoval. 2001. Extensión del límite sur para
Nothofagus glauca (Phil.) Krasser. Gayana Botanica 58(2): 139-142.

Martínez-Alonso C, F Valladares, J Camarero, M López Arias, M Serrano, J Rodríguez. 2007. The uncoupling of secondary growth, cone and litter production by intradecadal climatic variability in a mediterranean scots pine forest. Forest Ecology and Management 253: 19-29.

MMA (Ministerio de Medio Ambiente, CL). 2011. $7^{\circ}$ Proceso de Clasificación de Especies. Decreto Supremo No42/2011 del Ministerio de Medio Ambiente. Consultado 21 nov. 2017. Disponible en http://www.mma.gob.cl/clasificacionespecies/Anexo_septimoproceso/DS_42ApruebaCdeE_Septimo_Diario_Oficial.pdf

Ngao J, D Epron, C Brechet, A Granier. 2005. Estimating the contribution of leaf litter decomposition to soil $\mathrm{CO}_{2}$ efflux in a beech forest using ${ }^{13} \mathrm{C}$-depleted litter. Global Change Biology 11: 1768-1776.

Palacios-Bianchi P. 2002. Producción y descomposición de hojarasca en un bosque Maulino fragmentado. Seminario de Título. Santiago, Chile. Facultad de Ciencias, Universidad de Chile. 19 p.

Stolpe N, P Undurraga. 2016. Long term climatic trends in Chile and effects on soil moisture and temperature regimes. Chilean Journal of Agricultural Research 76(4): 487-496.

Venegas-González A, F Roig, A Gutiérrez, M Tomazello. 2018. Recent radial growth decline in response to increased drought conditions in the northernmost Nothofagus populations from South America. Forest Ecology and Management 409: 94-104.

Vilà M, J Vayreda, C Gracia, J Ibáñez. 2004. Biodiversity correlates with regional patterns of forest litter pools. Oecologia 139: 641-646.

Wutzler T, M Mund. 2007. Modelling mean above and below ground litter production based on yield tables. Silva Fennica 41(3): 559-574. 
Anexo 1. Composición promedio del compartimento resto de la hojarasca producida en un renoval pre andino de Nothofagus glauca de la región del Maule.

Mean composition [ $\left.\mathrm{g} \mathrm{m}^{-2} \mathrm{year}^{-1}\right]$ of the rest compartment of litter produced in a young pre-Andean Nothofagus glauca forest of Maule Region.

\begin{tabular}{|c|c|c|c|}
\hline Especie & $\mathrm{H}^{*}$ & $\mathrm{O} * *$ & {$\left[\mathrm{~g} \mathrm{~m}^{-2} \mathrm{año}^{-1}\right]$} \\
\hline Acrisione denticulata (Hook. et Arn.) B. Nord & $\mathrm{P}$ & $\mathrm{E}$ & $0,475 \pm 0,407$ \\
\hline Aextoxicon punctatum Ruiz et Pav. & $\mathrm{P}$ & $\mathrm{N}$ & $0,048 \pm 0,068$ \\
\hline Aristotelia chilensis (Molina) Stuntz & I & $\mathrm{N}$ & $1,750 \pm 1,765$ \\
\hline Azara integrifolia Ruiz et Pav. & $\mathrm{P}$ & $\mathrm{E}$ & $1,516 \pm 1,831$ \\
\hline Boquila trifoliolata (DC.) Decne. & $\mathrm{P}$ & $\mathrm{N}$ & $1,065 \pm 1,506$ \\
\hline Colliguaja salicifolia Gillies et Hook & $\mathrm{P}$ & $\mathrm{E}$ & $0,025 \pm 0,046$ \\
\hline Crataegus monogyna Jacq. & $\mathrm{P}$ & I & $0,003 \pm 0,010$ \\
\hline Cryptocarya alba (Molina) Looser & $\mathrm{P}$ & $\mathrm{E}$ & $5,106 \pm 6,377$ \\
\hline Cynanchum pachyphyllum (Decne.) K. Schum. & $\mathrm{P}$ & E & $0,007 \pm 0,015$ \\
\hline Escallonia pulverulenta (Ruiz et Pav.) Pers. & $\mathrm{P}$ & $\mathrm{E}$ & $2,298 \pm 2,199$ \\
\hline Gaultheria mucronata (L.f.) Hook. et Arn. & $\mathrm{P}$ & $\mathrm{N}$ & $0,044 \pm 0,122$ \\
\hline Hydrangea serratifolia (Hook. et Arn.) F. Phil. & $P$ & $\mathrm{~N}$ & $0,005 \pm 0,015$ \\
\hline Kageneckia oblonga Ruiz et Pav. & $P$ & $\mathrm{E}$ & $0,055 \pm 0,082$ \\
\hline Lapageria rosea Ruiz et Pav. & $P$ & $\mathrm{E}$ & $0,364 \pm 0,450$ \\
\hline Lardizabala biternata Ruiz et Pav. & $P$ & $\mathrm{E}$ & $1,025 \pm 0,902$ \\
\hline Lithraea caustica (Molina) Hook. et Arn. & $\mathrm{P}$ & $\mathrm{E}$ & $2,124 \pm 3,350$ \\
\hline Lomatia dentata (Ruiz et Pav.) R. Br. & $\mathrm{P}$ & $\mathrm{N}$ & $0,193 \pm 0,272$ \\
\hline Lomatia hirsuta (Lam.) Diels & $\mathrm{P}$ & $\mathrm{N}$ & $0,095 \pm 0,153$ \\
\hline Luma apiculata (DC.) Burret & $\mathrm{P}$ & $\mathrm{N}$ & $2,174 \pm 2,184$ \\
\hline Maytenus boaria Molina & $\mathrm{P}$ & $\mathrm{N}$ & $0,040 \pm 0,084$ \\
\hline Muehlenbeckia hastulata (Sm.) I.M. Johnst. & $\mathrm{P}$ & $\mathrm{N}$ & $0,021 \pm 0,050$ \\
\hline Myrceugenia obtusa (DC.) O. Berg & $\mathrm{P}$ & $\mathrm{E}$ & $4,364 \pm 3,656$ \\
\hline Nothofagus glauca (Phil.) Krasser & $\mathrm{C}$ & $\mathrm{E}$ & $0,362 \pm 0,654$ \\
\hline Nothofagus obliqua (Mirb.) Oerst. & $\mathrm{C}$ & $\mathrm{N}$ & $0,465 \pm 1,471$ \\
\hline Peumus boldus Molina & $\mathrm{P}$ & $\mathrm{E}$ & $3,090 \pm 3,069$ \\
\hline Pinus radiata D. Don & $\mathrm{P}$ & I & $1,224 \pm 1,494$ \\
\hline Quillaja saponaria Molina & $\mathrm{P}$ & $\mathrm{E}$ & $0,215 \pm 0,191$ \\
\hline Ribes punctatum Ruiz et Pav. & $\mathrm{P}$ & $\mathrm{N}$ & $0,004 \pm 0,009$ \\
\hline Schinus polygamus (Cav.) Cabrera & $\mathrm{P}$ & $\mathrm{N}$ & $0,003 \pm 0,008$ \\
\hline Sophora macrocarpa $\mathrm{Sm}$. & $\mathrm{P}$ & E & $0,046 \pm 0,031$ \\
\hline Tristerix corymbosus (L.) Kuijt & $\mathrm{P}$ & $\mathrm{N}$ & $0,121 \pm 0,143$ \\
\hline Material no identificado & & & $10,822 \pm 7,854$ \\
\hline
\end{tabular}

$\mathrm{H}^{*}$ : carácter de la hoja: C: caduca; I: invierno-verde; P: perenne.

$\mathrm{O}^{* *}$ : origen geográfico de la especie: N: nativa; E: endémica; I: introducida.

$\mathrm{H}$ *: leaf character: C: deciduous; I: winter green; P: perennial.

$\mathrm{O} * *$ : species geographical origin: $\mathrm{N}$ : native; E: endemic; I: introduced. 